\title{
Determinantes de la probabilidad de padecer enfermedades inflamatorias intestinales
}

\author{
Por Harold VÁsquez • Howard VÁsquez*
}

\begin{abstract}
Resumen
En base a un universo de 116 pacientes, realizamos un estudio observacional descriptivo transversal y de recolección de datos prospectivo con el fin de determinar la eficacia de la calprotectina fecal en pacientes conocidos y con alta sospecha de enfermedad inflamatoria del intestino. A diferencia de estudios anteriores, utilizamos una serie de modelos econométricos para estimar la probabilidad de que un paciente padezca Colitis Ulcerativa o Enfermedad del Crohn (CU-EC) dado el nivel de calprotectina detectado en las heces fecales. Los resultados indican que esta probabilidad puede diferir en magnitudes significativas dependiendo del sexo, la edad y los hábitos de consumo del paciente. Por ejemplo, en el caso de los hombres, la probabilidad de padecer CU-EC es significativamente mayor que para las mujeres, incluso en niveles bajos de calprotectina fecal. Además, la razón de probabilidades de padecer CU-EC aumenta hasta que el paciente alcanza los 37 años, edad en que la probabilidad de contraer una enfermedad inflamatoria intestinal comienza a reducirse paulatinamente. Finalmente, la probabilidad de que un paciente padezca $C U$ EC es mayor para las personas que declaran ser fumadoras de tabaco, a pesar de que este último resultado no aparenta ser significativo en términos estadísticos.
\end{abstract}

Palabras clave: calprotectina; colitis ulcerativa; enfermedad Crohn; Probit; Logit.

\footnotetext{
* Vásquez, Harold: Instituto Tecnológico de Santo Domingo (INTEC) y Banco Central de la República Dominicana. Dirección: Apartado Postal 1347, Av. Pedro H. Ureña esq. Leopoldo Navarro, Santo Domingo, República Dominicana. T: +1 809221 9111, (e-mail: harold.vasquez@intec.edu.do). Vásquez, Howard: Centro de Diagnósticos y Medicina Avanzada, Conferencias Médicas y Telemedicina (CEDIMAT) y Hospital Central de las Fuerzas Armadas (HCFFAA). Dirección: Departamento Enfermedades Inflamatoria Intestinal, CEDIMAT, Calle Pepillo Salcedo, esq Ortega y Gasset, Santo Domingo, República Dominicana Agradecimientos: agradezco el apoyo y dedicación de mi mentor, Dr. Sócrates Bautista, y agradezco al departamento de Gestión del Conocimiento, el Departamento de Investigaciones Científicas y el Comité de Ética de CEDIMAT. (e-mail: howardrvasquez@gmail.com).
} 


\title{
Determinants of probability of suffering inflammatory bowel diseases
}

\author{
By Harold VÁsquez • Howard VÁsquez*
}

\begin{abstract}
Based on a universe of 116 patients, we conducted a cross-sectional and prospective data collection descriptive observational study to determine the effectiveness of fecal calprotectin in known and highly suspected inflammatory bowel disease patients. Unlike previous studies, we use a series of econometric to estimate the probability that a patient suffering from ulcerative colitis or Crohn's disease (CU-EC) given the level of calprotectin detected in the stool models. The results indicate that this probability may differ in significant quantities depending on sex, age and habits of the patient. For example, in the case of men, the likelihood of developing CU-EC is significantly higher than for women, even at low levels of fecal calprotectin. In addition, the odds ratio of developing CU-EC increased until the patient reaches age 37, age at which the chance of developing inflammatory bowel disease begins to gradually decline. Finally, the probability that a patient has UC-EC is higher for people who say they smoke to snuff, even though this result seems not be significant in statistical terms.
\end{abstract}

Keywords: calprotectin; ulcerative colitis; Crohn's disease; Probit; Logit models

Códigos JEL: I10, I12, C25

\footnotetext{
* Vasquez, Harold: Instituto Tecnológico de Santo Domingo (INTEC) and Central Bank of the Dominican Republic. Address: Apartado Postal 1347, Av. Pedro H. Ureña esq. Leopoldo Navarro, Santo Domingo, Dominican Republic. T: +1 809221 9111, (e-mail: harold.vasquez@intec.edu.do). Vásquez, Howard: Centro de Diagnósticos y Medicina Avanzada, Conferencias Médicas y Telemedicina (CEDIMAT) y Hospital Central de las Fuerzas Armadas (HCFFAA). Address: Departamento Enfermedades Inflamatoria Intestinal, CEDIMAT, Calle Pepillo Salcedo, esq Ortega y Gasset, Santo Domingo, Dominican Republic. Acknowledgements: I am thankful for the support and dedication of my mentor, Dr. Sócrates Bautista, and I thank the Department of Knowledge Management, the Department of Scientific Research and the Ethics Committee of CEDIMAT.

(e-mail: howardrvasquez@gmail.com).
} 


\section{Introducción}

La categoría de enfermedad inflamatoria intestinal (EII) comprende trastornos caracterizados por una tendencia a la activación inmunitaria crónica o recidivante en el tracto gastrointestinal. La Colitis Ulcerosa (CU) y la enfermedad de Crohn (EC) representan las dos formas principales de la enfermedad inflamatoria intestinal.

La incidencia anual de la CU es de 24,3 por cada 100.000 personas por año en Europa; 6,3 por cada 100.000 personas por año en Asia y el Medio Oriente; y 19,2 por cada 100.000 personas por año en América del Norte. Mientras, en el caso de la EC, la incidencia anual estimada es de 12,7, de 5,0, y 20,2 por cada 100.000 personas en Europa, Asia y el Medio Oriente, respectivamente (Molodecky et al. 2012). Se ha demostrado a su vez que estas enfermedades poseen cierta agregación familiar, pues aproximadamente el $20 \%$ de los pacientes tienen algún familiar afectado. Otras evidencias sugieren que el pico máximo de incidencia ocurre entre los 15 y 35 años.

Históricamente, el diagnóstico de las EII se ha basado en una combinación de la historia clínica y la exploración, los parámetros sanguíneos, radiología y endoscopia. Esto causa que las EII sean diagnosticada de forma tardía y no manejadas a tiempo, debido en ocasiones al temor del método de diagnóstico invasivo y/o sus costos, los cuales pueden ser muy significativos para las personas de bajo ingreso. Por esto, la adición de un biomarcador fecal, como la prueba de calprotectina, capaz de reducir la necesidad de procedimientos endoscópicos invasivos, o la exposición a la radiación, además de ventajosa puede ahorrar significativos recursos tanto a agentes privados, empresas aseguradoras, como al sistema público de salud.

En este estudio analizamos la utilidad de la calprotectina fecal para diferenciar entre procesos inflamatorios intestinales y aquellos que no presentan enfermedad orgánica, de modo que también pueda servir como marcador predictivo de las EII con el fin de disminuir la 
cantidad de indicaciones innecesarias de estudios endoscópicos. ${ }^{1}$ Dentro de las ventajas de la calprotectina fecal podemos destacar que posee una sensibilidad de un $83 \%$ y especificidad de un $100 \%$ al momento de discriminar con otras enfermedades orgánicas. Varios estudios soportan su uso como instrumento para la diferenciación y establecer el grado de severidad de las EII en los pacientes. La amplia diversidad de dispositivos endoscópicos disponibles permite ampliar su aplicación diagnóstica y terapéutica.

El universo de este estudio estuvo representado por 116 pacientes y la muestra resultó en un total de 59 pacientes con alta sospecha y aquellos que ya eran conocidos con diagnóstico de enfermedad inflamatoria intestinal. Un $82 \%$ presentó aumento de la calprotectina fecal por encima del valor límite superior $(120 \mathrm{ug} / \mathrm{g})$; de este, un $88 \%$ se diagnosticó con enfermedad inflamatoria intestinal en comparación con el $12 \%$ restante que tenía un diagnóstico diferente.

A diferencia de las investigaciones realizadas al momento, nuestro enfoque está basado en la estimación de una serie de modelos econométricos para determinar la probabilidad de que un paciente padezca de Colitis Ulcerativa o Enfermedad de Crohn (CU-EC) dado el nivel de calprotectina detectado en las heces fecales. La ventaja de aplicar estos modelos es que nos permiten controlar por características observadas de los pacientes, como la edad, el sexo y los hábitos de consumo de los pacientes, que pueden incidir de manera significativa en la posibilidad de padecer una enfermedad inflamatoria intestinal.

Los resultados de los modelos de probabilidad lineal, Probit y Logit estimados indican que podemos predecir de forma eficiente la probabilidad de que un paciente padezca CU-EC a partir del resultado de la prueba de calprotectina fecal. Además, esta probabilidad puede diferir en magnitudes significativas dependiendo tanto de las caracte-

\footnotetext{
${ }^{1}$ La calprotectina fecal es un biomarcador que permite valorar la actividad inflamatoria intestinal en pacientes con colitis ulcerosa y con enfermedad de Crohn.
} 
rísticas del paciente como de sus hábitos de consumo. Por ejemplo, en el caso de los hombres, la probabilidad de padecer CU-EC es significativamente mayor que para las mujeres, incluso en niveles bajos de calprotectina fecal. Un hallazgo interesante es que la razón de probabilidades aumenta con la edad hasta que el paciente alcanza los 37 años, edad en que la probabilidad de contraer una enfermedad inflamatoria intestinal comienza a reducirse paulatinamente. Finalmente, la probabilidad de que un paciente padezca CU-EC es mayor para las personas que declaran ser fumadoras de tabaco, a pesar de que este último resultado no aparenta ser significativo en términos estadísticos.

\section{Antecedentes de la Calprotectina como Prueba para Detectar EII}

Descrita en 1980, la calprotectina es una proteína que se encuentra en el citosol de los neutrófilos y macrófagos compuestas de dos subunidades de las proteínas recombinantes humanas S100A8 y S100A9. Se puede detectar en el plasma, la orina, el líquido cefalorraquídeo, las heces, la saliva, el líquido sinovial y las biopsias de colon. Es estable en las heces de hasta siete días a temperatura ambiente y tiene una distribución homogénea en estas, propiedades que le confieren a las muestras fecales in situ (Fagerhol et al. 1980, Johne et al. 1997).

Desde 1992 se demostró la presencia de niveles elevados de calprotectina fecal en pacientes con CU y EC. Esto se debe a que los niveles de calprotectina en heces se correlacionan con la excreción fecal de leucocitos marcados, considerados como el estándar de oro para medir la inflamación intestinal. Varios estudios han investigado el valor de la calprotectina fecal en distinguir entre patología gastrointestinal orgánica y no orgánica en pacientes sintomáticos. ${ }^{2}$

\footnotetext{
2 Inicialmente la concentración de calprotectina fecal se reportó en miligramos por litro $(\mathrm{mg} / \mathrm{l})$, pero los ensayos más recientes indican la concentración de calprotectina fecal como microgramo por gramo $(\mathrm{mg} / \mathrm{g})$. En lo que concierne a este estudio utilizaremos la medición en base a $\mathrm{mg} / \mathrm{g}$.
} 
En un estudio reciente realizado por la unidad de gastroenterología del Western General Hospital en Edinburgh, con una población de 16,267 pacientes que presentaban signos y síntomas de enfermedad inflamatoria, Nicholas, Clark y Walkden (2014) encontraron que la calprotectina fecal fue significativamente mayor en los pacientes diagnosticados de EII (mediana calprotectina fecal $1251 \mathrm{mg} / \mathrm{g}$, rangos entre $532-2325 \mathrm{mg} / \mathrm{g}$ ) que aquellos con otras condiciones asociadas con un tracto gastrointestinal anormal (mediana calprotectina fecal $50 \mathrm{mg} / \mathrm{g}$, rangos entre $20-145 \mathrm{mg} / \mathrm{g}$ ) o con un diagnóstico funcional (mediana calprotectina fecal $20 \mathrm{mg} / \mathrm{g}$, rangos entre $20-50 \mathrm{mg} / \mathrm{g}$ ).

A su vez, estos investigadores también encontraron que los síntomas de alarma estaban presentes en el 25\% (140/566) de los pacientes diagnosticados con enfermedad funcional, el 86\% (78/91) de los pacientes diagnosticados con EII y el 54\% (35/65) de los diagnosticados con otra condición asociada con un tracto gastrointestinal anormal. El valor predictivo positivo (VPP) de los síntomas de alarma para la EII o un tracto gastrointestinal anormal vs enfermedad funcional fue de 0,45 (Intervalo de Confianza (IC) $95 \%$ 0,38 a 0,51), y el valor predictivo negativo fue de $0,91(0,88-0,93)$ con una sensibilidad de $0,72(0,65$ a 0,79$)$ y la especificidad de $0,75(0,71-0,79)$. Para la predicción de la EII, en comparación con la enfermedad funcional, el VPP fue de $0,36(0,29$ a 0,43$)$ y el VPN fue de $0,97(0,95-0,98)$, con una sensibilidad de $0,86(0,76$ a $0,92)$ y especificidad de $0,75(0,71-, 79)$ (ver Nicholas et al. 2014).

Niveles consistentemente elevados de calprotectina fecal han sido reportados en ambos pacientes: adultos y pediátricos, con EII en comparación con los pacientes con síndrome de intestino irritable o controles sanos. Por ejemplo, Van Rheenen et al. (2010), en un excelente meta análisis, comparó la precisión diagnóstica de la calprotectina fecal en la evaluación de pacientes con sospecha de EII. Se incluyeron trece estudios que resumen los datos de 1.041 pacientes (670 adultos, 371 niños). Los estudios fueron seleccionados por su robustez metodológica y tuvo que presentar un diseño pareado donde se midieron los valores de calprotectina fecal antes de la endoscopia. La sensibi- 
lidad y especificidad de las pruebas de las tasas de calprotectina eran 93\% y 96\%, respectivamente. En los adultos, el uso de la calprotectina fecal como prueba diagnóstica ante la sospecha de EII para decidir sobre la endoscopia se tradujo en una reducción del $67 \%$ en pacientes que requieren endoscopia, sino que también dieron lugar a un retraso en el diagnóstico de un $6 \%$ de los pacientes debido a resultados falsos negativos.

En los últimos años, el creciente interés en el valor de calprotectina en heces para el seguimiento de la enfermedad ha llevado a una serie de estudios que investigan su correlación con el grado de actividad de la EII, tal como se mide por endoscopia e histología. En esa línea, Schoepfer et al. (2010) reportaron resultados similares en pacientes con CU. Específicamente, utilizando el índice de Rachmilewitz, la actividad de la enfermedad endoscópica correlaciona mejor con la calprotectina fecal $(\mathrm{R}=0,834)$, seguido por el Índice de Actividad Clínica $(\mathrm{R}=0,672) .{ }^{3}$ La precisión global de calprotectina en la detección de la enfermedad por vía endoscópica activa fue del 89\%, y fue el único marcador para discriminar enfermedad inactiva, leve, moderada y altamente activa.

En otro estudio, Sipponen y Kolho (2010) investigaron 77 pacientes con EC que se sometieron a colonoscopia, y se comparó la actividad de la enfermedad endoscópica, como anotado por Índice de Severidad de la Enfermedad de Crohn (ISEC), con un índice de actividad clínico (IAC). La Calprotectina fecal se correlacionó bien con ISEC $(\mathrm{R}=0,729)$ y tenía una sensibilidad del $70 \%$ y una especificidad del $92 \%$ en la predicción de la enfermedad endoscópicamente activa, mientras que la sensibilidad del IAC era solo el $27 \%$, pero con mayor especificidad (94\%). En general, los valores de calprotectina fecal se correlacionan mejor con los hallazgos endoscópicos que con la actividad clínica. En consecuencia, este marcador sensible puede

\footnotetext{
3 El índice de Rachmilewitz (R) mide la correlación entre el nivel de calprotectina y el grado de actividad de la enfermedad endoscópica.
} 
detectar la actividad inflamatoria residual en pacientes con enfermedad presumiblemente exacerbada.

El curso natural de la EII se caracteriza típicamente por episodios recurrentes de recaída de la enfermedad con exacerbación y remisiones de la inflamación intestinal. Sin embargo, el curso de la enfermedad puede variar considerablemente. Recientemente, Kallel et al. (2010) estudiaron 53 pacientes con EC en remisión clínica, excluyendo específicamente los pacientes con EC limitada a intestino delgado. En un periodo de 12 meses de seguimiento, el 18\% desarrolló recaída clínica. Los valores de calprotectina fueron mayores en el grupo de recaída (381 mg /g vs $155 \mathrm{~g} / \mathrm{g}$, respectivamente). El uso de $340 \mathrm{mg} / \mathrm{g}$ como punto de corte, la calprotectina fecal proporcionó una sensibilidad del $80 \%$ y una especificidad del $91 \%$ en la predicción de la recaída clínica, lo que correspondía a un aumento del riesgo 18 veces de lo esperado.

En conclusión, existe una amplia evidencia que correlaciona de manera significativa los niveles elevados de calprotectina con el padecimiento de una enfermedad inflamatoria intestinal. Sin embargo, los estudios realizados al momento no han podido determinar en qué medida puede utilizarse la calprotectina fecal como un predictor eficiente de las enfermedades inflamatorias intestinales, como la CU y la EC. Más aun, tampoco se ha podido establecer cómo puede variar la eficiencia predictiva de la calprotectina fecal atendiendo a las características observadas de los pacientes y sus hábitos de consumo. El objetivo principal del presente estudio es contribuir en llenar estos vacíos que aún permanecen en la ciencia médica.

\section{Diseño Metodológico del Estudio y Técnica de Procedimiento}

Se realizó un estudio observacional descriptivo transversal y de recolección de datos prospectivo con el fin de determinar la utilidad de la calprotectina fecal en pacientes conocidos y con alta sospecha de enfermedad inflamatoria del intestino en el Centro de Diagnósticos 
y Medicina Avanzada, Conferencias Médicas y Telemedicina (CEDIMAT), en la ciudad de Santo Domingo, República Dominicana, en el periodo febrero-julio, 2015.

El universo estuvo representado por 116 pacientes que asistieron a la consulta del servicio de gastroenterología de CEDIMAT. La muestra resultó en un total de 59 pacientes con signos y síntomas de enfermedad y aquellos que ya eran conocidos con diagnóstico de enfermedad inflamatoria intestinal, que se le tomaron muestras de calprotectina fecal y fueron sometidos a una video gastroscopia o colonoscopia, representando un 50.86 por ciento.

Como criterio de inclusión fueron considerados los pacientes evaluados vía consulta por la clínica de enfermedades inflamatorias del servicio de gastroenterología de CEDIMAT que presentaron signos y síntomas de enfermedad inflamatoria intestinal y que son conocidos por padecer dicha enfermedad, durante el periodo febrero-julio, 2015. A su vez, quedaron excluidos los pacientes evaluados que no presentaron signos y síntomas de enfermedad inflamatoria intestinal y que no habían sido diagnosticados anteriormente. Se excluyeron además aquellos pacientes conocidos con Enfermedad Inflamatoria intestinal que no poseían un resultado de calprotectina fecal de base.

Con la autorización previa del Departamento de Gestión del Conocimiento, el Departamento de Investigaciones Científicas y el Comité de Ética de CEDIMAT, utilizamos un método analítico longitudinal de cohorte y de recolección de datos prospectivo, donde tomamos muestras biológicas y aplicamos un cuestionario, el cual consta de nueve acápites relacionados con las variables de los objetivos de esta investigación. De estos acápites cuatro fueron contestados por el paciente con preguntas cerradas y los cinco restantes fueron contestados por el entrevistador. Cada cuestionario tomó aproximadamente 5 minutos en ser contestado. Las muestras biológicas consistieron en la toma de las heces fecales de los sujetos entrevistados, para mediante la prueba de ELISA (enzimoinmunoanálisis por la técnica de ensayo por 
inmunoabsorción ligado a enzimas) determinar los niveles de calprotectina en cada toma. Para el levantamiento de las informaciones requeridas en el instrumento de recolección de datos, se utilizaron los expedientes clínicos de los usuarios. Todo esto con el previo consentimiento de cada paciente y de la institución. A cada paciente se le indicó que tiene el derecho de recibir información sobre los resultados de este estudio como beneficio que concierne a su estado de salud si así lo desea.

\section{Análisis de los Datos y Resultados Estadísticos}

Como mencionamos, de un total de 116 pacientes que asistieron a la consulta del servicio de gastroenterología con signos y síntomas de enfermedad inflamatoria intestinal de CEDIMAT, unos 59 correspondieron a pacientes de alta sospecha y otros que ya eran conocidos con diagnóstico de enfermedad inflamatoria intestinal, tomándoles así muestras de calprotectina fecal y fueron sometidos a una video gastroscopia o colonoscopia.

De los 59 pacientes evaluados vía consulta por la clínica de enfermedades inflamatorias del servicio de gastroenterología que presentaron signos y síntomas de enfermedad inflamatoria intestinal y que son conocidos por padecer dicha enfermedad, durante el periodo febrero-julio, 2015 , un $82 \%$ presentó aumento de la calprotectina fecal por encima del valor límite superior (120 ug/g); de este $82 \%$ (50 pacientes), un $88 \%$ (44 pacientes) se diagnosticó con enfermedad inflamatoria intestinal, presentando niveles de calprotectina fecal por encima de los $300 \mathrm{ug} / \mathrm{g}$, en comparación con el 12\% restante (6 pacientes), que tenía un diagnóstico diferente y cuyos niveles de calprotectina se encontraban leve o moderadamente aumentados (tabla 1). 
Tabla 1. Utilidad de la CALPROTECTINA FeCAL PARA Diferenciar ENTRE PROCESOS INFLAMATORIOS INTESTINALES Y AQUELLOS QUE NO PRESENTAN ENFERMEDAD ORGÁNICA.

\begin{tabular}{lcc}
\hline Patología & Pacientes & $\begin{array}{c}\text { Nivel Calprotectina Fecal } \\
\text { Promedio (ug/g) }\end{array}$ \\
\hline Colon Irritable & 6 & 14.5 \\
Colitis Ulcerativa & 23 & 464.6 \\
Enfermedad de Crohn & 21 & 311.4 \\
Enfermedad Celiaca & 1 & 0.8 \\
Mala Absorción & 1 & 135.0 \\
Gastritis Crónica & 2 & 284.5 \\
Gastritis Eritematosa & 1 & 58.6 \\
Helicobacter Pylori, Lupus & 1 & 21.0 \\
Sprue Tropical & 3 & 142.4 \\
Total & $\mathbf{5 9}$ & $\mathbf{3 1 4 . 0}$ \\
\hline
\end{tabular}

Fuente: elaborado por los autores a partir de los datos obtenidos en las entrevistas. Nota: $\mathrm{ug} / \mathrm{g}$ (miligramos de calprotectina/gramos de heces) con un rango de valor normal 0-120 ug/g. El 74.5\% de los pacientes presentó enfermedad inflamatoria intestinal mostrando los niveles promedios más elevados de calprotectina fecal.

La tabla 2 presenta informaciones relevantes sobre los pacientes que no eran conocidos en el centro y que fueron identificados con colitis ulcerativa o enfermedad de Crohn. Esta muestra incluye un total de 59 pacientes, de los cuales 28 corresponden a hombres y 31 a mujeres. A su vez, los análisis han detectado que 23 pacientes presentan Colitis Ulcerativa (CU), mientras que 21 pacientes reportan la Enfermedad de Crohn (EC). La CU parece ser más frecuente en mujeres (14 casos) que en hombres ( 9 casos). Lo contrario ocurre con la EC, la cual parecería ser más frecuente en hombres (13 casos) que en mujeres ( 8 casos).

En general, un total de 44 casos son identificados como positivos con Colitis Ulcerativa o Enfermedad de Crohn (CU-EC), los cuales estuvieron igualmente distribuidos entre hombres y mujeres. Entre los pacientes que padecen CU-EC sólo 6 personas reportaron ser consumidores habituales de bebidas alcohólicas, mientras 3 personas 
reportaron ser fumadores. En otras palabras, una amplia mayoría de las personas entrevistadas que resultaron como positivas con $\mathrm{CU}-\mathrm{EC}$ reportaron que no consumen alcohol ni tabaco. Los consumidores de café representan el $45 \%$ de la muestra, de los cuales unos 20 casos fueron identificados con CU-EC.

Tabla 2. Frecuencia de Casos Positivos y Negativos de Pacientes Identificados con Colitis Ulcerativa o Enfermedad de Crohn Según Sexo, Hábitos de Consumo, y Nivel Promedio de Calprotectina.

\begin{tabular}{|c|c|c|c|c|}
\hline Sexo / Hábitos & Negativo & Positivo & Calprotectina $^{1}$ & Total \\
\hline & \multicolumn{4}{|c|}{ Colitis Ulcerativa } \\
\hline Mujer & 17 & 14 & 344.54 & 31 \\
\hline \multirow[t]{2}{*}{ Hombre } & 19 & 9 & 280.12 & 28 \\
\hline & \multicolumn{4}{|c|}{ Enfermedad del Crohn } \\
\hline Mujer & 23 & 8 & 344.54 & 31 \\
\hline \multirow[t]{2}{*}{ Hombre } & 15 & 13 & 280.12 & 28 \\
\hline & \multicolumn{4}{|c|}{ Colitis y/o Enfermedad Crohn } \\
\hline Mujer & 9 & 22 & 344.54 & 31 \\
\hline Hombre & 6 & 22 & 280.12 & 28 \\
\hline Consume Alcohol & 5 & 6 & 207.75 & 11 \\
\hline $\begin{array}{l}\text { No Consume } \\
\text { Alcohol }\end{array}$ & 10 & 38 & 338.31 & 48 \\
\hline Fuma Tabaco & 1 & 3 & 255.73 & 4 \\
\hline No Fuma Tabaco & 14 & 41 & 318.21 & 55 \\
\hline Consume Café & 7 & 20 & 360.66 & 27 \\
\hline $\begin{array}{l}\text { No Consume } \\
\text { Café }\end{array}$ & 8 & 24 & 274.58 & 32 \\
\hline
\end{tabular}

Fuente: elaborado por los autores con datos de la encuesta. ${ }^{1}$ Nivel promedio de calprotectina en ug/g.

La gráfica 1 muestra el nivel promedio de calprotectina fecal (CF) acorde al sexo y los hábitos de consumo de los pacientes muestreados. En general, los pacientes femeninos presentan un nivel promedio de CF mayor $(344.5 \mathrm{ug} / \mathrm{g})$ al de los masculinos $(280.1 \mathrm{ug} / \mathrm{g})$. 
A su vez, los pacientes que reportaron ser consumidores de alcohol y tabaco presentaron niveles promedio de CF de $207.7 \mathrm{mg} / \mathrm{g}$ y 255.7 $\mathrm{mg} / \mathrm{g}$, respectivamente, los cuales son inferiores a los niveles promedio reportados por no consumidores de dichos productos. En el caso de los consumidores de café, la media reportada de la prueba de CF fue $(360.7 \mathrm{mg} / \mathrm{g})$ superior a la media reportada por los no tomadores de café $(274.6 \mathrm{mg} / \mathrm{g})$. Sin embargo, a pesar de las diferencias observadas, los resultados de la prueba t-Student para diferencia de medias indican que las diferencias en las medias de CF entre no son estadísticamente significativas para ninguna de las categorías analizadas. ${ }^{4}$

\section{Gráfica 1. Nivel Promedio de Calprotectina Fecal Según Sexo y Hábitos de Consumo}

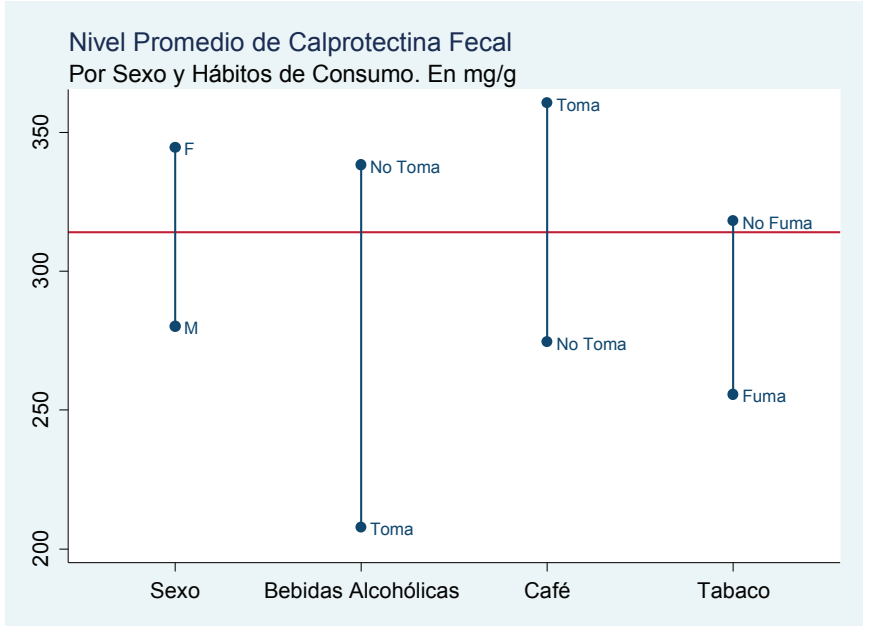

Se realizó una correlación entre los niveles de calprotectina y la respuesta a los diferentes tipos de tratamientos utilizando los índices de TrueLove y Harvey, con el fin de demostrar si existe una relación entre tratamiento, dosis y respuestas. En ambos casos, tanto en

\footnotetext{
${ }^{4}$ Como mencionamos, para probar la diferencia de las medias entre los pacientes acorde a su sexo y hábitos de consumo, utilizamos la prueba t-Student de diferencia de medias. Esta prueba fue realizada en ambos casos considerando si la varianza del nivel de calprotectina difiere o no entre las categorías mencionadas. El ajuste de varianzas se realizó aplicando la fórmula de Welch. En todos los casos, no encontramos diferencias estadísticamente significativas en las medias de los grupos.
} 
pacientes con Colitis Ulcerativa como en pacientes con Enfermedad de Crohn, no se pudo encontrar dicha relación, ya que pacientes que presentaban iguales tratamientos a igual dosificación mostraban distintos índices de actividad, por lo que podemos concluir que simplemente hay pacientes que se acoplan mientras otros muestran resistencia a los diferentes tipos de tratamientos (Tabla 3A y 3B).

Tabla 3A. Utilidad de la CALPRotectina feCAl EN PaCientes diagnosticados CON ENFERMEDAD INFLAMATORIA INTESTINAL Y CORRELACIONARLOS CON LA RESPUESTA AL TRATAMIENTO.

\begin{tabular}{lr}
\hline I. TrueLove & Cambio Promedio \\
\hline Abandonado & 225.9 \\
$\quad$ Severo & 225.9 \\
Azatioprina 1.5gr & 146.2 \\
Severo & 146.2 \\
Azatioprina 1gr & -11.5 \\
Moderado & -11.5 \\
Pentasa 2gr & 78.4 \\
Leve & -64.6 \\
Moderado & 266.0 \\
Severo & 43.0 \\
Pentasa 2gr + Pentasa 1gr Sup & 166.5 \\
Severo & 166.5 \\
Pentasa 4gr & 151.5 \\
Leve & -68.9 \\
Moderado & 189.0 \\
Severo & 223.2 \\
Pentasa 4gr + Pentasa 1gr Sup & 585.4 \\
Moderado & 585.4 \\
Pentasa sup & -85.7 \\
Leve & -85.7 \\
Total & 142.4 \\
\hline
\end{tabular}

Fuente: realizada por el autor a partir de los datos obtenidos en las entrevistas. Nota: el cambio promedio es una diferencia porcentual entre los niveles de calprotectina antes y después de llevar los tratamientos antes descritos. 
TABLa 3B. Utilidad de La CALPROTECTINA FeCAL EN PACIENTES DiAgnosticados CON ENFERMEDAD INFLAMATORIA INTESTINAL Y SU CORRELACIÓN CON LA RESPUESTA AL TRATAMIENTO.

\begin{tabular}{lc}
\hline I. Harvey & Cambio Promedio \\
\hline Pentasa 2gr & -52.2 \\
Actividad Moderada & -52.2 \\
Azatriopina 1.5gr & -34.8 \\
Remisión & -34.8 \\
Azatioprina 2gr & 125.3 \\
Actividad Severa & 125.3 \\
Azatioprina 1gr + Adalinumab & -69.0 \\
Actividad Moderada & -55.2 \\
Remisión & -96.5 \\
Azatioprina 1gr & -36.0 \\
Actividad Moderada & -1.0 \\
Remisión & -88.6 \\
Azatioprina 1.5gr & -50.4 \\
Actividad Moderada & -50.4 \\
Azatioprina 0.5gr & -74.5 \\
Actividad Moderada & -74.5 \\
Adalinumab & 5.0 \\
Actividad Moderada & 5.0 \\
Abandonado & -93.3 \\
Remisión & -93.3 \\
Total & -39.0 \\
\hline
\end{tabular}

Fuente: realizada por el autor a partir de los datos obtenidos en las entrevistas. Nota: el cambio promedio es una diferencia porcentual entre los niveles de calprotectina antes y después de llevar los tratamientos antes descritos.

Por otro lado, se quiso comprobar la utilidad de la calprotectina fecal como marcador para predecir un estudio endoscópico patológico y correlacionar sus valores con el grado de actividad en la enfermedad inflamatoria intestinal. En cuanto a predecir un estudio endoscópico patológico se pudo observar que del total de pacientes que presentaban algún signo y/o síntoma de enfermedad inflamatoria intestinal y 
que a su vez tenían valores elevados de calprotectina fecal, el $88 \%$ presentó un estudio endoscópico patológico mientras que el 12\% restante presentó un estudio endoscópico normal. La tabla 4 muestra la relación entre los valores de la calprotectina fecal con el grado de actividad en la enfermedad inflamatoria intestinal en base al índice de mayo, el cual reportó un aumento promedio de la calprotectina fecal en aquellos pacientes con un índice moderado y severo de $596.6 \mathrm{ug} / \mathrm{g}$ y $548.8 \mathrm{ug} / \mathrm{g}$, respectivamente; por otra parte, los pacientes con índice leve solo presentaron un aumento promedio de la calprotectina fecal de $245.2 \mathrm{ug} / \mathrm{g}$.

TABla 4. Utilidad de la CALPROTECTINA FECAL COMO MARCADOR PARA PREDECIR UN ESTUDIO ENDOSCÓPICO PATOLÓGICO Y CORRELACIONAR SUS VALORES CON EL GRADO DE ACTIVIDAD EN LA ENFERMEDAD INFLAMATORIA INTESTINAL.

\begin{tabular}{lrr}
\hline I. Mayo & Calprotectina Fecal Promedio & Pacientes \\
\hline Leve & 245.2 & 13.0 \\
Moderado & 596.6 & 21.0 \\
Severo & 548.8 & 2.0 \\
Total & 467.0 & 36.0 \\
\hline
\end{tabular}

Fuente: realizada por el autor a partir de los datos obtenidos en las entrevistas de pacientes con colitis ulcerativa. Entre los 36 pacientes que presentaron estudio endoscópico patológico, los mayores niveles de calprotectina fecal se encontraban en aquellos que reportaban un índice moderado y severo.

\section{Resultados de los Modelos de Probabilidad}

La tabla 5A muestra los resultados de las estimaciones en base al Modelo de Probabilidad Lineal (MPL), y los modelos Probit y Logit. Los parámetros de los modelos probit y logit han sido escalados para hacerlos comparables con los parámetros del MPL. Los estadísticos R2 y Pseudo-R2, en todos los casos, superan el $40 \%$, lo que sugiere un buen ajuste de los modelos estimados. Esto se confirma cuando calculamos el porcentaje correctamente predicho (\%), el cual mide en qué porcentaje el modelo predice la variable resultado o la proba- 
bilidad de que un paciente padezca de CU ó EC. En ambos modelos, Probit y Logit, el porcentaje de predicción supera el $80 \%$, lo cual es una magnitud importante.

Tabla 5A. Estimaciones de los Modelos de Probabilidad Lineal, Probit y Logit.

\begin{tabular}{lccc}
\hline \multirow{2}{*}{ Edad } & MPL & Probit & Logit \\
& 0.0298 & 0.0172 & 0.016 \\
Edad $^{2}$ & {$[0.0191]$} & {$[0.0163]$} & {$[0.0187]$} \\
& -0.0004 & -0.0002 & -0.0002 \\
Masculino & {$[0.0002]^{*}$} & {$[0.0002]$} & {$[0.0002]$} \\
& 0.1509 & 0.2321 & 0.2384 \\
Alcohol & {$[0.1076]$} & {$[0.1215]^{*}$} & {$[0.1359]^{*}$} \\
& -0.2958 & -0.2556 & -0.2767 \\
Cafe & {$[0.1420]^{* *}$} & {$[0.131]^{*}$} & {$[0.1439]^{*}$} \\
Tabaco & -0.0775 & -0.2161 & -0.2377 \\
& {$[0.1108]$} & {$[0.1096]^{* *}$} & {$[0.1267]^{*}$} \\
Nivel de CF & 0.11 & 0.0609 & 0.0844 \\
& {$[0.2150]$} & {$[0.2158]$} & {$[0.227]$} \\
Constante & 0.0005 & 0.0012 & 0.0015 \\
& {$[0.0002]^{* * *}$} & {$[0.0004]^{* * *}$} & {$[0.0006]^{* * *}$} \\
$R^{2} /$ Pseudo- $R^{2}$ & 0.1287 & -0.3535 & -0.3423 \\
$R . V$. & {$[0.3874]$} & {$[0.3218]$} & {$[0.3706]$} \\
\% CP & 0.45 & 0.42 & 0.43 \\
$N$ & 0.0000 & 0.0003 & 0.0002 \\
\hline \hline
\end{tabular}

Nota. *Nivel de significancia al 10\%, ** al 5\%, y*** al 1\%. MPL: Modelo de robabilidad Lineal. CF: Calprotectina Fecal. Para el MPL el estadístico es $R^{2}$. Para los modelos Probit y Logit es estadístico es Pseudo- $R^{2}$. R.V.: razón de verosimilitud (p-valor). \%CP: Porcentaje Correctamente Predicho.

Las estimaciones de la tabla $5 \mathrm{~A}$ muestran resultados interesantes. Primero, el nivel de calprotectina fecal (CF) está relacionado positiva y significativamente con la probabilidad de padecer CU-EC. Cabe destacar que el nivel de significancia del parámetro estimado es superior al $1 \%$, lo que indica que la prueba de CF es un buen predictor 
de la probabilidad de que un paciente padezca de colitis ulcerativa o enfermedad de Chron.

El resto de los resultados indican que la probabilidad de padecer CU-EC es mayor para los hombres, en comparación con las mujeres, al igual que para los pacientes que reportan ser fumadores. Por otro lado, la probabilidad de padecer CU-EC es significativamente menor para los pacientes que reportan ser consumidores de café y consumidores ocasionales de alcohol. El coeficiente positivo en la variable $E d a d$, y negativo para $E d a d^{2}$, sugiere que la probabilidad de que una persona padezca CU-EC aumenta con la edad cuando se es joven, pero luego llega un momento en la persona adulta que dicha probabilidad comienza a descender según se envejece. Específicamente, la probabilidad de padecer CU ó EC aumenta hasta que el paciente alcanza a los 37 años, edad en la cual dicha probabilidad comienza a descender. Sin embargo, a pesar de su importancia, debemos señalar que este último resultado no es estadísticamente significativo.

La tabla 5B presenta el resultado del modelo probabilístico logístico. Los coeficientes estimados representan el cambio en la razón de probabilidades (en inglés, "odds ratio") de que un paciente padezca CU-EC con respecto a la posibilidad de que no padezca esta enfermedad. Cuando la razón de probabilidades es igual a uno significa que las posibilidades de poseer la enfermedad son las mismas que no poseerla ante un cambio en los regresores o variables independientes. Es decir, en este caso, los regresores no afectan significativamente la razón de probabilidad de poseer la enfermedad. La tabla 5B también indica que el modelo de la columna 6 (M6) es que presenta un mejor resultado de predicción. Específicamente, el M6 predice correctamente la probabilidad de que el paciente padezca CU-EC en casi un $90 \%$. La tabla $5 \mathrm{C}$, mostrada más adelante, presenta los indicadores de bondad de ajuste de los modelos M1 al M6, y la misma también sugiere que el modelo M6 presenta las mejores medidas de ajuste basadas en los criterios de información Akaike (AIC), de Bayes (BIC) y $\mathrm{R}^{2}$-ajustado. 
Tabla 5B. Regresión Logística: Ratios de Riesgo Relativo (RRR)

\begin{tabular}{|c|c|c|c|c|c|c|}
\hline $\begin{array}{l}\text { Regre- } \\
\text { sores }\end{array}$ & M1 & M2 & M3 & M4 & M5 & M6 \\
\hline \multirow[t]{2}{*}{ Edad } & 1.1253 & 1.1371 & 1.2357 & 1.2442 & 1.2502 & 1.1438 \\
\hline & {$[0.1307]$} & [0.1321] & {$[0.1617]$} & [0.1668] & {$[0.1707]$} & [0.1795] \\
\hline \multirow[t]{2}{*}{$E_{d a d^{2}}$} & 0.9983 & 0.9982 & 0.9972 & 0.9971 & 0.997 & 0.9983 \\
\hline & [0.0013] & [0.0013] & {$[0.0015]^{*}$} & {$[0.0015]^{*}$} & {$[0.0015]^{*}$} & [0.0018] \\
\hline \multirow[t]{2}{*}{ Hombre } & & 1.6045 & 2.4448 & 2.5817 & 2.4409 & 7.4007 \\
\hline & & [1.0562] & [1.8229] & [1.9877] & [1.9047] & {$[8.4415]^{*}$} \\
\hline \multirow[t]{2}{*}{ Alcohol } & & & 0.1469 & 0.1368 & 0.1195 & 0.098 \\
\hline & & & {$[0.1262]^{\star *}$} & {$[0.1219]^{\star *}$} & {$[0.1098]^{\star *}$} & {$[0.1183]^{*}$} \\
\hline \multirow[t]{2}{*}{ Café } & & & & 0.7912 & 0.8365 & 0.1359 \\
\hline & & & & [0.5802] & [0.6187] & {$[0.1445]^{*}$} \\
\hline \multirow[t]{2}{*}{ Tabaco } & & & & & 2.752 & 2.0314 \\
\hline & & & & & [3.8995] & [3.8707] \\
\hline \multirow[t]{2}{*}{$\begin{array}{l}\text { Nivel de } \\
\text { CF }\end{array}$} & & & & & & 1.0125 \\
\hline & & & & & & {$[0.0047]^{* * *}$} \\
\hline Pseudo $R^{2}$ & 0.0866 & 0.0944 & 0.1732 & 0.1747 & 0.1829 & 0.4291 \\
\hline $\begin{array}{l}\% \text { Predi- } \\
\text { cho }\end{array}$ & $74.58 \%$ & $74.58 \%$ & $72.88 \%$ & $72.88 \%$ & $76.27 \%$ & $89.83 \%$ \\
\hline$N$ & 59 & 59 & 59 & 59 & 59 & 59 \\
\hline
\end{tabular}

Notas. Nivel de significancia del $* \mathrm{p}<0.1 ; * * \mathrm{p}<0.05 ; * * * \mathrm{p}<0.01$. CF: Calprotectina Fecal. Edad2: edad al cuadrado.

Las estimaciones de la tabla 5B indican que la posibilidad de padecer CU-OC es siete veces mayor cuando el paciente es hombre. El coeficiente de la variable Hombre (7.4007) es estadísticamente significativo a un nivel de confianza del 10\%. Por otro lado, y quizás contrario a lo que pudiéramos esperar, la razón de posibilidades de poseer CU-EC se reducen cuando el paciente consume de forma casual alcohol o café. Para los pacientes fumadores de tabaco, las posibilidades de padecer CU-EC es dos veces mayor con relación la posibilidad de padecer dicha enfermedad cuando no se es fumador.

Los coeficientes en las variables $E d a d$ y $E d a d^{2}$ indican que las posibilidades de padecer CU-EC aumentan con la edad cuando el 
paciente es joven, pero esta razón de probabilidades se reduce según la persona envejece. Ya anteriormente habíamos indicado que la edad en que la razón de probabilidades de padecer CU-EC comienza a reducirse es cuando la persona tiene 37 años. Finalmente, cuando el nivel de calprotectina fecal del paciente incrementa en 1 ug/g, la posibilidad de que el paciente padezca de CU-EC aumenta en .0125 más que las posibilidades de no poseer dicha enfermedad. Este último resultado es estadísticamente significativo a un nivel de confianza del $1 \%$.

En ocasiones, en vez de utilizar las razones de probabilidades, se hace más fácil interpretar las estimaciones de los modelos logísticos en términos de los efectos marginales que tienen los regresores sobre las probabilidades, mediante la estimación de los efectos marginales promedios. La tabla 5D presenta estos resultados. En este caso, los resultados indican que los pacientes de sexo masculino tienen una probabilidad entre .21 y .23 mayor de padecer CU-EC en comparación con los pacientes femeninos. De igual forma, por cada aumento de 10 años en la edad del paciente, la probabilidad de padecer CU-EC incrementa entre 0.14 y 0.17 . A su vez, el aumento de $100 \mathrm{ug} / \mathrm{g}$ en el nivel de calprotectina incrementa la probabilidad de padecer CU-EC entre 0.12 y 0.13 puntos. 
Tabla 5C. Medidas de Ajuste de la Regresión Logística

\begin{tabular}{lcccccc}
\hline \multicolumn{1}{c}{ Indicadores } & $\mathrm{M} 1$ & $\mathrm{M} 2$ & $\mathrm{M} 3$ & $\mathrm{M} 4$ & $\mathrm{M} 5$ & $\mathrm{M} 6$ \\
\hline $\mathrm{N}$ : & 59 & 59 & 59 & 59 & 59 & 59 \\
Log-Lik Intercept Only & -33.45 & -33.45 & -33.45 & -33.45 & -33.45 & -33.45 \\
Log-Lik Full Model & -30.553 & -30.291 & -27.658 & -27.607 & -27.333 & -19.097 \\
$D$ & $61.106(56)$ & $60.581(55)$ & $55.315(54)$ & $55.213(53)$ & $54.666(52)$ & $38.195(51)$ \\
LR & $5.793(2)$ & $6.318(3)$ & $11.584(4)$ & $11.686(5)$ & $12.233(6)$ & $28.704(7)$ \\
Prob > LR & 0.055 & 0.097 & 0.021 & 0.039 & 0.057 & 0 \\
McFadden's R2 & 0.087 & 0.094 & 0.173 & 0.175 & 0.183 & 0.429 \\
McFadden's Adj R2 & -0.003 & -0.025 & 0.024 & -0.005 & -0.026 & 0.19 \\
ML (Cox-Snell) R2 & 0.094 & 0.102 & 0.178 & 0.18 & 0.187 & 0.385 \\
Cragg-Uhler(Nagelk- & 0.138 & 0.15 & 0.263 & 0.265 & 0.276 & 0.568 \\
erke) $R^{2}$ & & & & & & \\
McKelvey \& Zavoina's & 0.13 & 0.141 & 0.27 & 0.273 & 0.293 & 0.843 \\
R2 & 0.109 & 0.113 & 0.183 & 0.186 & 0.193 & 0.488 \\
Efron's R2 & 3.781 & 3.828 & 4.508 & 4.526 & 4.656 & 20.923 \\
Variance of ${ }^{*}$ & 3.29 & 3.29 & 3.29 & 3.29 & 3.29 & 3.29 \\
Variance of error & 0.746 & 0.746 & 0.729 & 0.729 & 0.763 & 0.898 \\
Count R2 & 0 & 0 & -0.067 & -0.067 & 0.067 & 0.6 \\
Adj Count R2 & 1.137 & 1.162 & 1.107 & 1.139 & 1.164 & 0.919 \\
AIC & 67.106 & 68.581 & 65.315 & 67.213 & 68.666 & 54.195 \\
AlC*n & -167.236 & -163.683 & -164.872 & -160.896 & -157.366 & -169.76 \\
BIC & 2.362 & 5.914 & 4.726 & 8.702 & 12.232 & -0.162 \\
BIC' & 73.338 & 76.891 & 75.703 & 79.678 & 83.209 & 70.815 \\
BIC used by Stata & 67.106 & 68.581 & 65.315 & 67.213 & 68.666 & 54.195 \\
AIC used by Stata & & & & & \\
\hline
\end{tabular}

Nota. Los indicadores mostrados para M1 hasta M6 corresponden a los modelos (1) al (6) estimados en la tabla 5B. AIC: Información de Criterio de Akaike. BIC: Información de criterio de Bayes. 
Tabla 5D. Efecto Marginal Promedio

\begin{tabular}{lcc}
\hline & \multicolumn{2}{c}{ Efectos Marginales } \\
\hline \multirow{2}{*}{ Edad } & Logit & Probit \\
& 0.014 & 0.0172 \\
Edad ${ }^{2}$ & {$[0.0159]$} & {$[0.0154]$} \\
& -0.0002 & -0.0002 \\
Hombre & {$[0.0002]$} & {$[0.0002]$} \\
& 0.2092 & 0.2321 \\
Alcohol & {$[0.1057]^{* *}$} & {$[0.1039]^{* *}$} \\
& -0.2428 & -0.2556 \\
Café & {$[0.1078]^{* *}$} & {$[0.1127]^{* *}$} \\
& -0.2086 & -0.2161 \\
Tabaco & {$[0.0941]^{* *}$} & {$[0.0943]^{* *}$} \\
& 0.0741 & 0.0609 \\
Calprotectina & {$[0.1983]$} & {$[0.2156]$} \\
$N$ & 0.0013 & 0.0012 \\
${ }^{*} p<0.1 ;{ }^{* *} p<0.05 ;{ }^{* * *} p<0.01$. & {$[0.0003]^{* * *}$} & {$[0.0003]^{* * *}$} \\
\hline
\end{tabular}

Finalizando, el resultado de las estimaciones y las pruebas realizadas sugieren que el modelo puede predecir de forma muy eficiente la probabilidad de que un paciente padezca CU-EC dado el nivel de calprotectina detectado en las heces fecales. Al mismo tiempo, esta probabilidad puede diferir en magnitudes muy significativas dependiendo del sexo del paciente. Las gráficas a continuación muestran las predicciones de las probabilidades de padecer CU-EC dado el nivel de calprotectina fecal del paciente. En el caso de los hombres, las probabilidades son significativamente más altas que para las mujeres, incluso en niveles bajos de calprotectina fecal. Las estimaciones indican que para un nivel de calprotectina de $100 \mathrm{ug} / \mathrm{g}$ la probabilidad 
de padecer CU-EC es de 0.39 en las mujeres, 0.74 en los hombres, y 0.55 en el caso de ambos sexos. Esta probabilidad puede aumentar significativamente hasta 0.81 para las mujeres, 0.95 para los hombres, y 0.89 en ambos sexos si los pacientes presentan niveles de calprotectina fecal de $300 \mathrm{ug} / \mathrm{g}$. A niveles de calprotectina superiores a los 500 ug/g la probabilidad de que una persona padezca CU-EC es aproximadamente de del 1 ó $100 \%$.

Gráfica 2A. Probabilidad de Padecer Colitis Ulcerativa o Enfermedad de Crohn Acorde al Nivel de Calprotectina del Paciente. Grupo de Todos los PACIENTES.

Probabilidad Estimada de Padecer Colitis Ulcerativa o Enfermedad del Crohn Según el nivel de calprotectina del paciente (en ug/g)

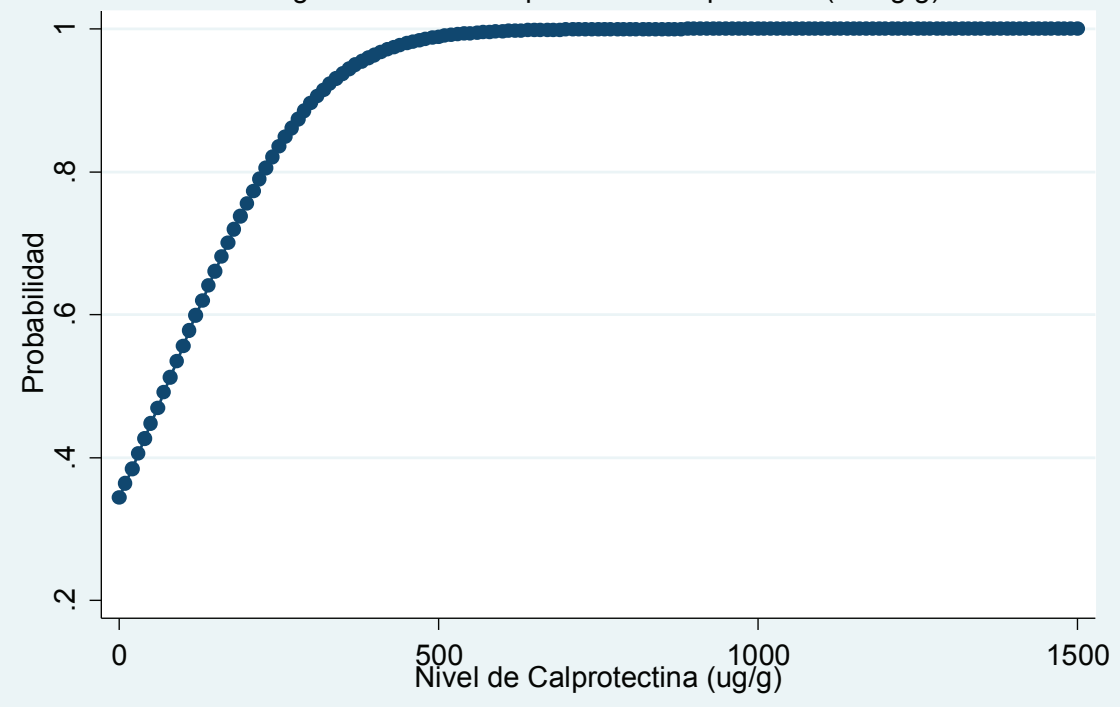


Gráfica 2B. Probabilidad Estimada de Padecer Colitis Ulcerativa o Enfermedad de Crohn, Acorde al Nivel de Calprotectina. Estimación por Grupos Según Sexo.

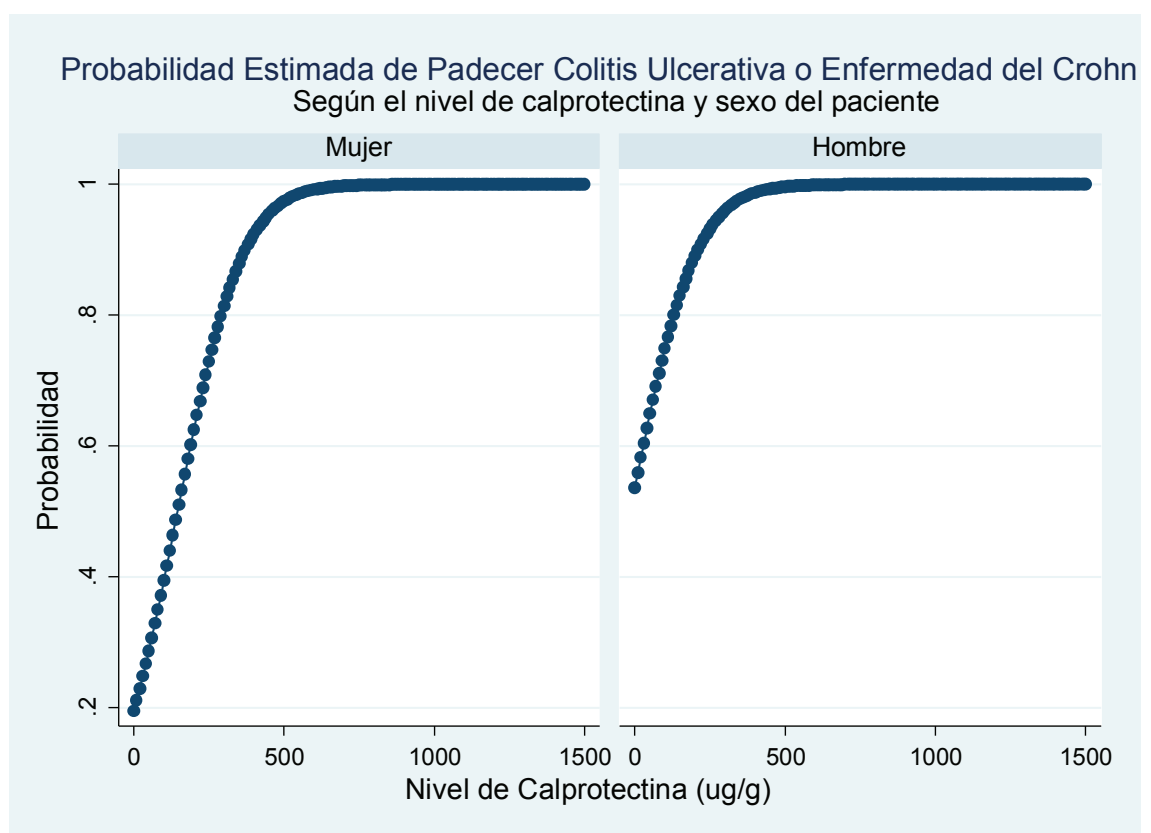

VI. Conclusiones y Recomendaciones

De los 59 pacientes evaluados vía consulta por la clínica de enfermedades inflamatorias del servicio de gastroenterología que presentaron signos y síntomas de enfermedad inflamatoria intestinal y que son conocidos por padecer dicha enfermedad, durante el periodo febrero-julio, 2015, un $82 \%$ presentó aumento de la calprotectina fecal por encima del valor límite superior (120 ug/g); de este 82\% (50 pacientes), un $88 \%$ (44 pacientes) se diagnosticó con enfermedad inflamatoria intestinal, presentando niveles de calprotectina fecal por encima de los $300 \mathrm{ug} / \mathrm{g}$, en comparación con el 12\% restante (6 pacientes) que tenía un diagnóstico diferente y cuyos niveles de calprotectina se encontraban leve o moderadamente aumentados. 
De los 44 pacientes que fueron diagnosticados con enfermedad inflamatoria intestinal, el 100\% presentó niveles elevados de calprotectina fecal; por otro lado, de los 15 pacientes que presentaban iguales signos y/o síntomas pero que tenían un diagnóstico diferente, el 40\% presentó niveles elevados de calprotectina fecal, mostrando así una sensibilidad de un $100 \%$ y una especificidad de un $60 \%$.

En aquellos pacientes que fueron diagnosticados con Colitis Ulcerativa o Enfermedad de Crohn se realizó una correlación entre los niveles de calprotectina y la respuesta a los diferentes tipos de tratamientos con el fin de demostrar si existe una relación entre tratamientos, dosis y respuestas. Dicha relación no fue encontrada ya que pacientes que presentaban iguales tratamientos a igual dosificación mostraban distintos índices de actividad de la enfermedad.

En cuanto a predecir un estudio endoscópico patológico, se pudo observar que del total de pacientes que presentaban algún signo y/o síntoma de enfermedad inflamatoria intestinal y que a su vez tenían valores elevados de calprotectina fecal, el $88 \%$ presentó un estudio endoscópico patológico, mientras que el $12 \%$ restante presentó un estudio endoscópico normal.

Al comparar los valores de la calprotectina fecal con los diferentes grados de actividad en la enfermedad inflamatoria intestinal, se reportó un aumento promedio de la calprotectina fecal en aquellos pacientes con un índice moderado y severo de $596.6 \mathrm{ug} / \mathrm{g}$ y $548.8 \mathrm{ug} / \mathrm{g}$, respectivamente; por otra parte, los pacientes con índice leve solo presentaron un aumento promedio de la calprotectina fecal de $245.2 \mathrm{ug} / \mathrm{g}$.

Al demostrar la utilidad de la calprotectina fecal como marcador biológico predictivo en la evolución de los pacientes con enfermedad inflamatoria del intestino, se pudo observar, en el caso de los pacientes con Colitis Ulcerativa, que aquellos pacientes que se encontraban en actividad severa y moderada mostraron un aumento promedio de la calprotectina fecal de $182.3 \mathrm{ug} / \mathrm{g}$ y $227.9 \mathrm{ug} / \mathrm{g}$, 
respectivamente, mientras que aquellos que se encontraban en actividad leve presentaron una disminución promedio de la calprotectina fecal de $68.4 \mathrm{ug} / \mathrm{g}$.

En el caso de los pacientes con Enfermedad de Crohn, se observó que aquellos que se encontraban en actividad severa mostraron un aumento promedio de la calprotectina fecal de $125.3 \mathrm{ug} / \mathrm{g}$, mientras que aquellos que se encontraban en actividad moderada y remisión presentaron una disminución promedio de la calprotectina fecal de $36.3 \mathrm{ug} / \mathrm{g}$ y $72.7 \mathrm{ug} / \mathrm{g}$, respectivamente.

Los resultados de los modelos de probabilidad lineal, Probit y Logit indican que podemos predecir de forma muy eficiente la probabilidad de que un paciente padezca CU-EC dado el nivel de calprotectina detectado en las heces fecales.

Esta probabilidad puede diferir en magnitudes muy significativas dependiendo del sexo del paciente. En el caso de los hombres, las probabilidades de padecer Colitis Ulcerativa o Enfermedad de Crohn son significativamente más altas que para las mujeres, incluso en niveles bajos de calprotectina fecal. Por ejemplo, las estimaciones indican que para un nivel de calprotectina de $100 \mathrm{ug} / \mathrm{g}$ la probabilidad de padecer CU-EC es de 0.39 en las mujeres, 0.74 en los hombres, y 0.55 en el caso de ambos sexos. Esta probabilidad puede aumentar significativamente hasta 0.81 para las mujeres, 0.95 para los hombres, y 0.89 en ambos sexos si los pacientes presentan niveles de calprotectina fecal de $300 \mathrm{ug} / \mathrm{g}$. A niveles de calprotectina superiores a los 500 ug/g la probabilidad de que una persona padezca CU-EC es aproximadamente del 1 o 100\%. En promedio, el aumento de $100 \mathrm{ug} / \mathrm{g}$ en el nivel de calprotectina incrementa la probabilidad de padecer CU-EC entre 0.12 y 0.13 puntos.

Además, encontramos que las posibilidades de padecer CU-EC aumentan con la edad cuando el paciente es joven, pero esta razón de probabilidades se reduce según la persona envejece. Específicamente, 
la probabilidad de que un paciente padezca de CU-EC incrementa con la edad hasta que el paciente alcanza los 37 años, edad en que la probabilidad de contraer una enfermedad inflamatoria intestinal comienza a reducirse paulatinamente. Por ejemplo, cuando la edad de un paciente pasa de 20 a 30 años y a 40 años, la probabilidad de padecer CU-EC aumenta en 0.12 puntos, 0.10 puntos y 0.08 puntos, respectivamente.

Contrario quizás a lo que podría esperarse, la probabilidad de padecer una enfermedad inflamatoria intestinal, ya sea Colitis Ulcerativa o Enfermedad de Crohn, es significativamente menor para aquellos pacientes que declaran ser consumidores casuales de alcohol o café. Sin embargo, esta probabilidad es mayor para las personas que declaran ser consumidoras de tabaco, a pesar de que este último resultado no aparenta ser estadísticamente significativo.

Finalmente, en base a las conclusiones obtenidas en esta investigación se presentan las siguientes recomendaciones.

- Indicar una prueba de calprotectina fecal en todos aquellos pacientes con sospecha de enfermedad inflamatoria intestinal, aunque esta no posee una alta especificidad su alto nivel de sensibilidad podría por un lado descartarnos dicha patología u orientarnos a un diagnóstico más probable, a la vez que nos sirve como un valor base en la evolución de aquellos pacientes que cursen con la enfermedad.

- Realizar en el departamento de enfermedades inflamatoria intestinal un protocolo estricto y cronológico de tratamiento desde el inicio del diagnóstico, basado en la respuesta y evolución de cada paciente.

- Utilizar la calprotectina fecal como un estudio de seguimiento al menos 3 meses después de que el paciente haya iniciado un nuevo tratamiento, ya que este estudio ha demostrado tanto una 
importante disminución en aquellos pacientes que han respondido al tratamiento como un importante aumento en aquellos que han mostrado resistencia o abandono del mismo.

- Indicar el análisis de la calprotectina fecal junto a cada estudio endoscópico control, ya que la misma demostró una relación con los índices de actividad, además de poder orientarnos del seguimiento del paciente al compararla con una calprotectina fecal base al momento del diagnóstico y con un primer control luego del inicio de su último tratamiento.

\section{Referencias}

Fagerhol MK, Dale I, y Andersson T. 1980. “A radioimmunoassay for a granulocyte protein as a marker in studies on the turnover of such cells." Bull Eur Physiopathol Respir; 16 Suppl: 273-282.

Johne B, Fagerhol MK, Lyberg T, Prydz H, Brandtzaeg P, Naess-Andresen CF, y Dale I. 1997. "Functional and clinical aspects of the myelomonocyte protein calprotectin." Mol Pathol; 50: 113123.

Kallel L, Ayadi I, Matri S, Fekih M, Mahmoud NB, y Feki M. 2010. "Fecal calprotectin is a predictive marker of relapse in Crohn's disease involving the colon: a prospective study." Eur J Gastroenterol Hepatol; 22(3): 340-5.

Molodecky NA, Soon IS, Rabi DM, Ghali WA, Ferris M, Chernoff G. 2012. "Increasing incidence and prevalence of the inflammatory bowel diseases with time, based on systematic review." Gastroenterology; 42(1): 46-54.e42; enero 2012.

Nicholas A. Kennedy, Annalie Clark, y Andrew Walkden. 2014. "Clinical utility and diagnostic accuracy of faecal calprotectin for IBD at first presentation to gastroenterology services 
in adults aged 16-50 years". J Crohns Colitis; 9(1): 41-49. January 2012.

Schoepfer AM, Beglinger C, Straumann A, Trummler M, Vavricka SR, y Bruegger LE. 2010. "Fecal calprotectina correlates more closely with the Simple Endoscopic Score for Crohn's disease (SESCD) than CRP, blood leukocytes, and the CDAI." Am J Gastroenterol;105(1):162-9.

Sipponen T y Kolho KL. 2010. "Faecal calprotectin in children with clinically inflammatory bowel disease." Scand J Gastroenterol; 45(78): 872-7.

Van Rheenen PF, Van de Vijver E y Fidler V. 2010. "Faecal calprotectin for screening of patients with suspected inflammatory bowel disease: diagnostic metaanalysis." British Medical Journal; 341: c3369. 